\title{
The production and evaluation of biologically synthesized anticancer Chlorambucil - DTPA - Methionine
}

\author{
Elham Poonaki ${ }^{1}$, Leila Pishkar², Mahdyeh Zarandooz ${ }^{3}$, Hadi Hejazinia ${ }^{3}$, Marzyeh Hosseini ${ }^{4}$, \\ Artin Assadi ${ }^{3}$, Mohammad Zahedi ${ }^{3}$ and Shahin Hadadian ${ }^{* 5}$ \\ ${ }^{1}$ Department of Biotechnology, I.A.U of Damghan, Damghan, Iran \\ ${ }^{2}$ Young Researchers and Elite Club, Islamshahr Branch, Islamic Azad University, Islamshahr, Iran \\ ${ }^{3}$ Department of Radiopharmacy, Faculty of Pharmacy, Tehran University of Medical Sciences, Tehran, Iran \\ ${ }^{4}$ Department of Cellular and Molecular Biology, Islamic Azad University, Tehran North Branch,Tehran, Iran \\ ${ }^{5}$ Nanobiotechnology Department, Pasteur Institute of Iran, Tehran, Iran
}

\begin{abstract}
Chromabucil is a direct acting direct acicular anticancer drug which is still widely used in the treatment of some cancers as a primary treatment, but its use is often limited due to the unwanted side effects of this drug due to the lack of specificity in targeting cancer cells. In this project, our effort to increase the specificity of Chlorambucil using methionine amino acid has led to the production and evaluation of biological antimicrobial Nano conjugate Chlorambucil-DTPA-Methionine. Research has shown that the consumption and harvesting of cancer cells increases significantly over nitrogen and polyamide compounds, while some amino acids, including methionine amino acids, are more expressed on the surface of the cancer cells, resulting in tissue Cancer cells increase to methionine amino acid, so in our study, we have been using methionine amino acid as a carrier and enhancer of the uptake of Chlorambucil antimicrobial to produce this conjugate from the connector We used DTPA. After studying this Nano conjugate and examining its structure, we investigated the therapeutic and biological effects of this nanoagonergic drug as compared to Chlorambucil on the MCF-7 and HT-29 cell line (breast cancer and colon cancer), including The MTT assay tests the determination of cell death and cell necrosis and a test for the determination of conjugate toxicity on mice, which ultimately led to the understanding that the new nano conjugate Chlorambucil-DTPA-Methionine not only retained its anti-cancer properties against the Chlorambucil drug But has shown less abnormal toxicity.
\end{abstract}

KEY WORDS: BREAST CANCER / CHLORAMBUCIL / METHIONINE / DTPA / CONJUGATE

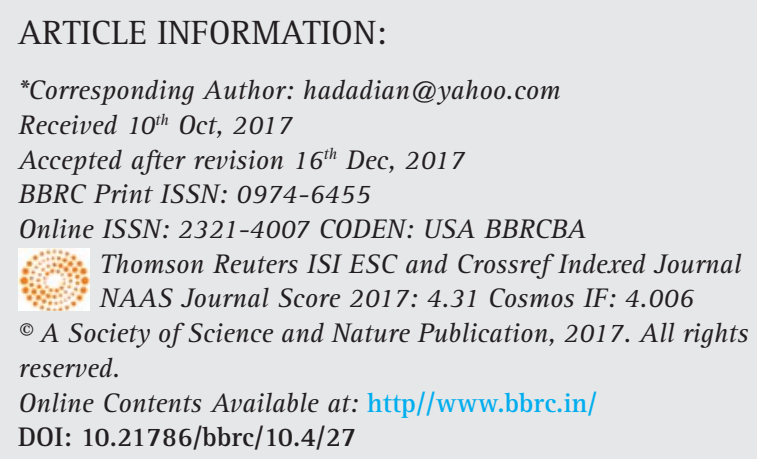




\section{INTRODUCTION}

Since cancer is a fatal illness worldwide, it is the second leading cause of death in the world after cardiovascular disease and, according to annual reports, causes many deaths from cancer. Today's treatments are very costly and have unpleasant side effects in the patient's body. Today, efforts are being made to find newer and more effective treatments, including these treatments, for chemical treatments. Nowadays, there is a lot of research to discover new drugs, appropriate drug delivery routes, and optimal drug targeting with less side effects. Recently, therapeutic studies have been conducted on the transmission of antibody-dependent drugs, but since the therapeutic method of drug delivery using antibodies also has inefficiencies and transitional problems, costs and side effects, in particular, itself, a newer research for the transfer of anticancer drugs is taking place. Since then, research has shown that the consumption and removal of cancer cells increases significantly over nitrogen and polyamide compounds, and the expression of some amino acids such as methionine amino acids on the surface of the cancer cells is more pronounced and therefore cancer tissue cells to increase the amino acid methionine, (Levine et al., 2000, Palmer et al. 2009, Roché et al. 2011).

In this study we have used methionine amino acid as carrier and antimicrobial agent for the treatment of Chlorambucil. Cancer treatment as an anticancer drug, like other anticancer drugs, has side effects on the cancerous patient's body, the reason is that by designing an anticancer drug conjugate using methionine amino acid (as an enhancement of cellular uptake and DTPA interface and conducting biologic studies of the effect of this conjugate on cancer cells through drug delivery to reduce side effects and Increase the efficacy of Chlorambucil to as an appropriate drug delivery method, we tried to do that. So far, hybrids have been made of noninterfacing Chlorambucil, such as Chlorambucil glucose and Chlorambucil -tyrosine (Gupta A et al. 2010).

But the proper interface for efficient drug delivery is very important. We have been working to produce this conjugate for the amino acid linkage of methionine We used the DTPA connector for Chlorambucil amine, which looks very good interface due to amine groups, because amine receptors from cancer cells increase and drug delivery will be more successful. We then performed the relevant biological tests on the MCF-7 and HT-29 cell line breast cancer cells.

\section{MATERIAL AND METHODS}

Following chemicals were used in the study.Chlorambucil (Sigma-Aldrich, USA) USA • Cell line MCF-7 and
HT-29 (Pasteue Institute of Tehran) • Sulfo-NHS (SigmaAldrich, USA) USA) • EDC (Sigma-Aldrich, USA) • PBS (Merck, Germany) • DMSO (Merck, Germany) • Sephadex G-10 Fine (Sigma-Aldrich) • Chloroform (Merck, Germany) • FCS (Seromed Biochrom, Germany) ) • FBS (Merck, Germany) • RPMI medium (Sigma-Aldrich, USA) - Penicillin powder (Sigma-Aldrich, USA) • Streptomycin powder (Sigma-Aldrich, USA) • The TNF-alpha kit (The RayBio ${ }^{\circledR}$ Human TNF -alpha ELISA) Kitin AnnexinV-PI (BD Pharmingen, UK).

Combination or hybridization of an antimicrobial agent of Chlorambucil and methionine amino acid, produced by the interface of DTPA herein. $2.320 \mathrm{~g}$ of methionine (if used $3 \mathrm{mg}$ of Chlorambucil) is added in 5-5 milliliters of water Soluble. 2. Add $1 \mathrm{mg}$ DTPA to the solution and dissolve it by sterilizer. 3. After 1 to 2 minutes, add twice as much amino acid as EDC to the previous solution. To the product of the first stage after 1 to 5 minutes of production, $758 \mathrm{mg} / \mathrm{kg}$ of Chlorambucil is added. 2. To complete the Chlorambucil dissolution, dissolve it with sterilization. 3. The product is ready for the next reaction. Use of DSMO is due to fatloving Chlorambucil (CBL) and DMSO helps to dissolve it and accelerate the reaction. At this stage, purification of the conjugated product is studied. The purity of the compound produced was investigated by TLC chromatography.

A 10 milliliter TLC solvent was prepared containing 3 milliliters of chloroform (nonpolar solvent to dissolve lipophilic compounds) and 7 milliliters of methanol (polar solvent to dissolve hydrophilic compounds) (30\% chloroform and 70\% methanol) TLC papers were cut in rectangles with dimensions, length $8-7 \mathrm{~cm}$ and width of 5-4 cm. Then 1 millimeter above the solvent line (the highest part of the paper that is placed inside the solvent), with the capillary tube, there were delicate spots spaced apart, which included, respectively, methionine, DTPA, Sulfur-NHS, conjugate synthesis They were Chlorambucil and EDC. Each paper was placed in a solvent containing a $45^{\circ}$ angle inside the container and due to the evaporation of chloroform and methanol, as well as the toxicity of chloroform, it should be closed in a container. The required amount of $25-26 \mathrm{C}^{\circ}$ and the required time in this method is about 20 minutes. Be Due to the fact that all materials and stains are colorless, UV light was used to observe the movement rate of the material, and stains were observed in the range of 280-220. The patches created with DTPA, sulfur-NHS, EDC, and CBL stain were a blurry and sharp spot. By this method, the result was that the synthesis product, conjugate Chlorambucil-methionine-DTPA, was present in the fourth to seventh test tubes.

Mass Spectroscopy analysis was carried out to confirm methionine - DTPA- Chlorambucil 
Conjugation. MTT is a colorimetric assay which is used to assess cell viability based on metabolic activity. This assay is based on reduction of yellow tetrazolium salt (MTT) to form dark colored formazan dye by dehydrogenase enzymes in metabolically active cells the cells (MCF7) and (HT-29) were brooded with different concentrations of methionine - DTPA- Chlorambucil (0.1, 0.2, $0.5 \mathrm{mg} / \mathrm{ml}$ and Chlorambucil $0.5 \mathrm{mg} / \mathrm{ml}$ and untreated cell (as a negative control) for $48 \mathrm{~h}$.. After incubation $(48 \mathrm{~h}), 50 \mu \mathrm{l}$ XTT detection solution was added to each well of 96-well plate and the plate is kept in the incubator for $2 \mathrm{~h}$. The formazan dye is soluble in aqueous solution and can be measured by evaluating the absorbance at $450 \mathrm{~nm}$ using a spectrophotometer. The results were compared to the untreated control cells. Statistical data analysis was done using Prism and excels software (Microsoft Office 2013). For quantitative data analysis paired One Way ANOVA in case of cluster comparison, were applied. $\mathrm{P}<0.05$ were considered statistically significant.

\section{RESULTS AND DISCUSSION}

In gel filtration chromatography, the solution was removed from the chromatography column with $1 \mathrm{ml}$ (20 drops) in 20 separate test tubes. Then, each tube was subjected to thin layer chromatography (TLC) to verify the accuracy of the final product. On the TLC paper, one millimeter above the solvent line with a capillary tube, delicate spots were separated from each other, which consisted of methionine, DTPA, Sulfo-NHS, synthetic conjugate, Chlorambucil-methionine- DTPA, Chlorambucil (CBL), and EDC. The stain was then investigated by the UV lamp and the presence of the product in the fourth to seventh tubes was determined, and then the $\mathrm{Rf}$ of each compound was calculated according to the formula given in Chapter 2, as follows (Table 1)

LC / MC mass spectrometry (LC / MC) spectroscopy was used to study the molecular structure and identify the conjugated Chlorambucil -methionine DTPA. The results are presented Figure 1.The 1983 molecular weight spectrum shows well that the molecular weight of 1983 is exactly equal to the conjugate molecular weight, which is proof of conjugated synthesis. The syntactic conjugate molecule is well visible

In Figure 2 and 3 the results of the compound toxicity on the MCF-7 and HT-29 cell line respectively indicate that our combination at $5 \mathrm{mg} / \mathrm{ml}$ dose has a significant toxicity $(p<0.05)$, and this toxicity is completely equal to Chlorambucil. Making targeted drugs is a very important part of cancer treatment. The use of non-cancerous anticancer drugs causes various side effects in the patient. One of the oldest anti-cancer drugs is Chlorambucil, which belongs to the group of alkalizing drugs, its effects on the cells are non-specific and mechanically unknown and have many side effects for patients. The production of a molecular conjugate of Chlorambucil -methionine was performed with the goal of having anticancer properties and also acting purposefully. The ability to kill Conjugate Chlorambucil-Methionine-

Table 1. The values of Rf are calculated from the TLC paper from the distance that the
material goes through to the distance that the solvent travels.
\begin{tabular}{|l|l|l|l|l|l|l|}
\hline Material & Methionine & DTPA & Sulfo-NHS & CBL-Met DTPA & CBL & EDC \\
\hline Rf & 72.0 & 82.0 & 49.0 & 40.0 & 32.0 & 88.0 \\
\hline
\end{tabular}

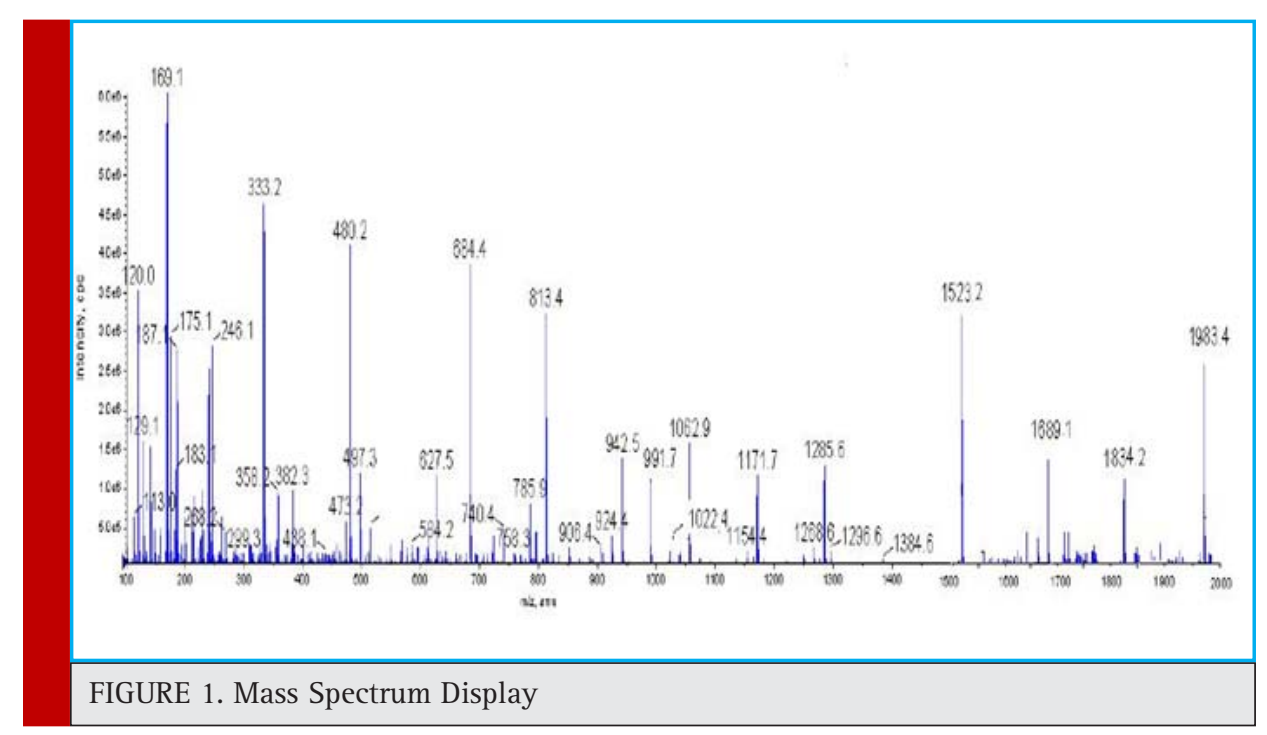




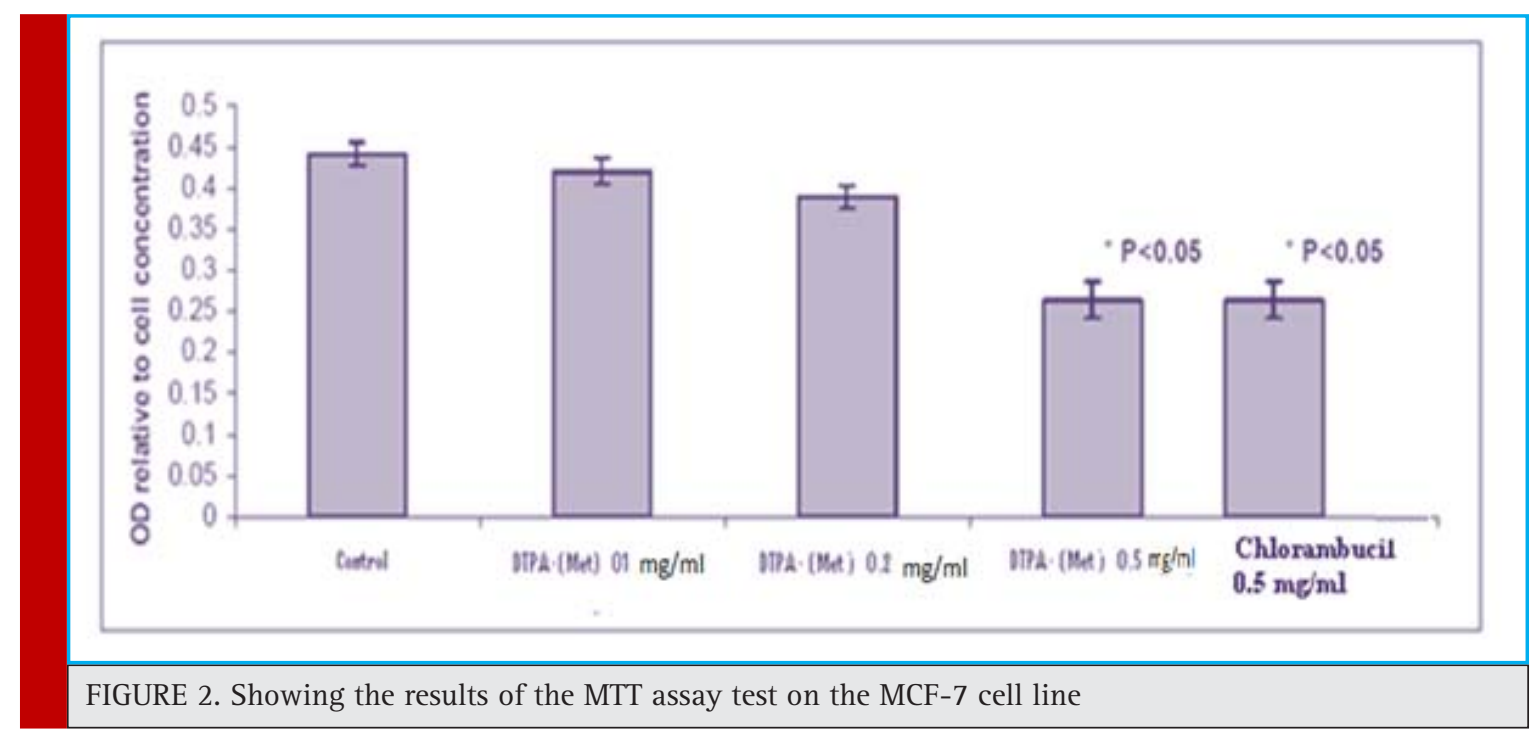

DTPA in similar doses compared to Chlorambucil on the HT-29 and MCF-7 cell line was higher.

According to the results of experiments on conjugate and its comparison with Chlorambucil, the results were that: the binding of methionine amino acid to Chlorambucil not only does not eliminate the anticancer effect of this drug, but also increases its anticancer effect and increases the conjugated effect Because cancer cells tend to receive high amounts of polyamine compounds, the binding of the methionine amino acid to Chlorambucil will result in a targeted formulation that can absorb cancer cells of this conjugate. In 2004, according to Bothenichen's research on polyamine and cancer, it was discovered that the accumulation of polyamide compounds in cancerous tissues increased, as well as the concentration of these compounds in the body fluids of patients with cancer, (Buchrach et al. 2004) and in 2005 researchers it was found that the transfer of some essential amino acids in cancer cells increases, because in cells with high metabolism, the absorption of amino acids also increases. Therefore, using drugs reduces the synthesis of polyamine or inhibits the carriers of these amino acids, it can inhibit cancer, and these drugs can be a good option for cancer treatment (Yoon et al. 2005). In 2009, Palmer and colleagues in their research stated that increasing the concentration of polyamines plays an important role in the development of cancer, from the onset to the maintenance of the phenotype of the transformation, (Palmer et al. 2009).

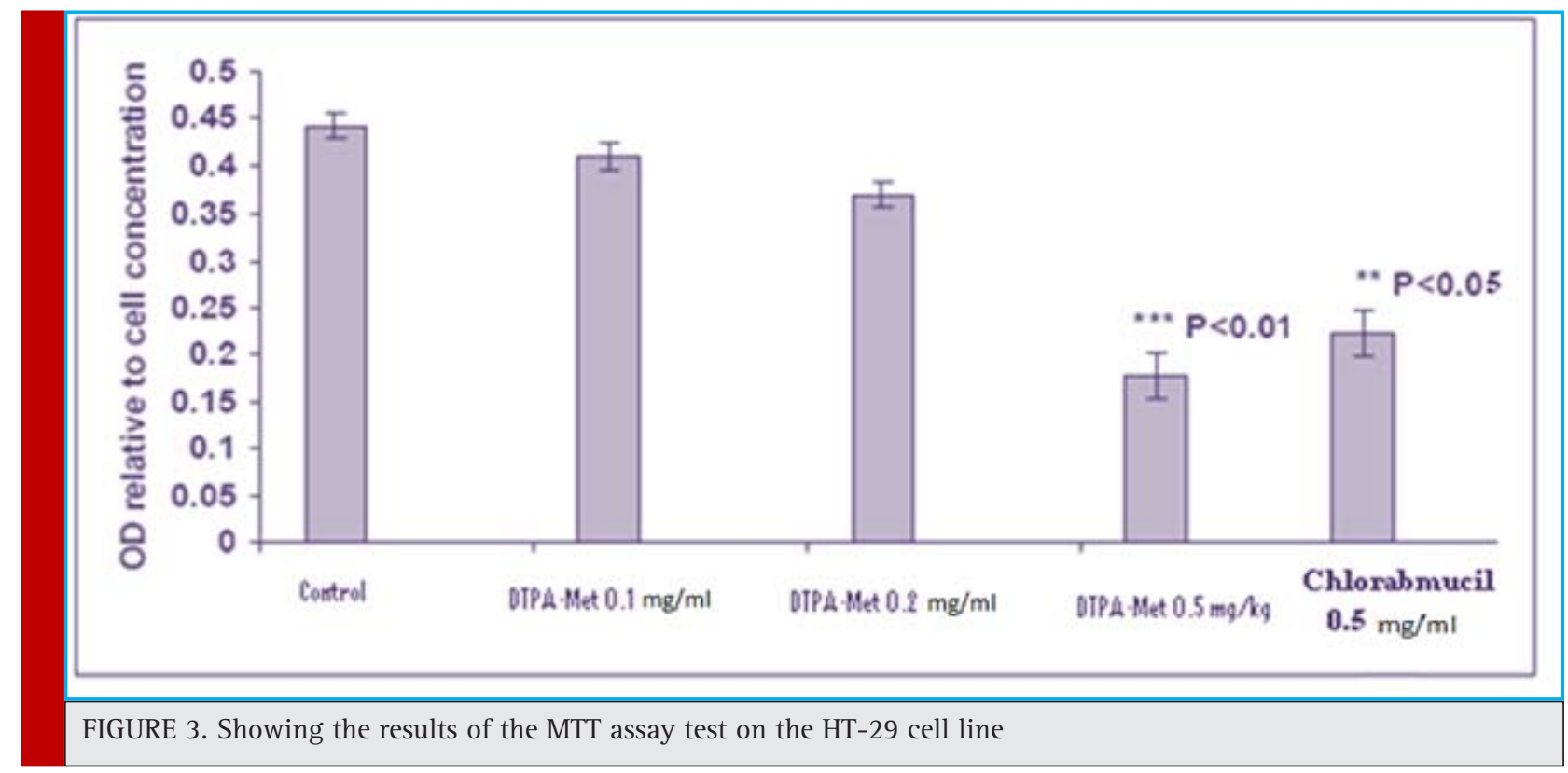


One of the ways of cancer cells to increase the concentration of polyamine compounds is the absorption of polyamines by the polyamine (PTS) transfer system in these cells. PTS is a rugged system that transmits a large amount of polyamines. Therefore, it may be possible to transfer cytotoxic drugs by binding to polyamine vesicles and using this system to target cancer cells selectively (Yoon et al. 2005). In 1997, the dependence of the growth of a number of solid tumors on high levels of methionine and the increased need for tumor cells to methionine were observed in relation to normal cells, which suggests that methionine can be considered as an appropriate choice for tumor treatment. (Buchrach et al. 2004). Conjugate Chlorambucil -methionine DTPA-D, due to its high chemical content, is considered as a polyamide compound and also has a methionine-like acid component in its structure, which is due to two reasons for its absorption in cancer cells. So far, much research has been done to transfer targeted drugs through specific molecules to tumors. The use of transfusion carriers and the manufacture of drug conjugates can have a more effective and specific effect on drugs. Due to the high side effects of Chlorambucil anticancer drugs and its nonspecific effects on the cells of the body, the purpose of this drug and its side effects have been many studies on Chlorambucil conjugation.

In 2010, they used Chlorambucil-estradiol for chemotherapy for breast cancer and found that they had better and better efficacy compared to Chlorambucil (Gupta et al. 2010). In 2011, researchers, given the frequent expression of receptors Folate (FRS) at the level of the malignant tumor cells synthesized two new conjugated folate carrying Chlorambucil, and they were biologically evaluated on the leukemia cell line. The results of the antitumor activity showed more conjugates compared to non-conjugated Chlorambucil (Guaragna et al. 2011).

By reviewing the articles, it was concluded that the synthesized conjugates of Chlorambucil had a positive effect and their effectiveness was much better than that of Chlorambucil, and these considerations made the conjugated Chlorambucil -DTPA-methionine II as a novel drug in The treatment of breast cancer has also been strengthened. Also, according to the aforementioned articles, the synthesis of conjugate Chlorambucil and the study of binding methods led to the conclusion that the binding of Chlorambucil to amino acids was carried out from its carboxyl side, not from the chlorine region. Because chlorine is a very important factor for killing cells by alkylating them. The carboxyl side of the Chlorambucil has a pharmacokinetic aspect (no dynamic aspect). Through this position, the drug enters the body, it traverses and excretes a pathway. Therefore, the manipulation and binding of the amino acid in this region, with the exception of the effect on absorption and its shelf-life in the body will not have an effect on inhibiting the effectiveness of the medicine.

\section{ACKNOWLEDGMENTS}

The authors would to thank Tehran University of Medical Sciences as well as all laboratories and technicians who provided supported during within the course of the study.

\section{CONFLICT OF INTEREST}

The authors declared no conflict of interest.

\section{REFERENCES}

Buchrach U. Polyamines and cancer: Mini review article. (2004) Amino Acids. 26(4), 307-9

Gupta A, Saha P, Descôteaux C, Leblanc, Asselin É, Bérubé G. (2010) Design, synthesis and biological evaluation of estradiol-chlorambucil hybrids as anticancer agents. Bioorganic \& Medicinal chemistry letters. 20(5), 1614-1618.

Gupta A, Saha P, Descôteaux C, Leblanc V, Asselin E, Bérubé G, 2010 Design, synthesis and biological evaluation of estradiolchlorambucil hybrids as anticancer agents. Bioorg Med Chem Lett. 2010 Mar 1;20(5):1614-8.

Levine RL, Moskovitz J, Stadtman ER. (2000) Oxidation of methionine in proteins: roles in antioxidant defense and cellular regulation. IUBMB Life. 50, 301-7.

Palmer AJ, Ghani RA, Kaur N, Phanstiel 0, Wallace HM. (2009) A putrescine-anthracene conjugate: a paradigm for selective drug delivery. Biochem J. 424(3), 431-8.

Roché H, Vahdat LT, (2011) Treatment of metastatic breast cancer: second line and beyond. Ann Oncol. 22(5), 1000-10.

Yoon JH, Kim IJ, Kim H, Kim HJ, Jeong MJ, Ahn SG, Kim SA, Lee CH, Choi BK, Kim JK, Jung KY, Lee S, Kanai Y, Endou H, Kim DK. (2005) Amino acid transport system L is differently expressed in human normal oral keratinocytes and human oral cancer cells. Cancer Lett. 222(2), 237-45. 